\title{
Identification of distinct physiochemical properties of the toxic prefibrillar species formed by $A \beta$ peptide variants
}

\author{
Anna-Lena Göransson, Daniel Kanmert, K. Peter R. Nilsson, \\ Katarina Kågedal and Ann-Christin Brorsson
}

\section{Linköping University Post Print}

N.B.: When citing this work, cite the original article.

Original Publication:

Anna-Lena Göransson, Daniel Kanmert, K. Peter R. Nilsson, Katarina Kågedal and AnnChristin Brorsson, Identification of distinct physiochemical properties of the toxic prefibrillar species formed by $\mathrm{A} \beta$ peptide variants, 2012, Biochemical and Biophysical Research Communications - BBRC, (420), 4, 895-900.

http://dx.doi.org/10.1016/j.bbrc.2012.03.097

Copyright: Elsevier

http://www.elsevier.com/

Postprint available at: Linköping University Electronic Press http://urn.kb.se/resolve?urn=urn:nbn:se:liu:diva-73185 
Identification of distinct physiochemical properties of toxic prefibrillar species formed by $A \beta$ peptide variants

Anna-Lena Göransson ${ }^{\mathrm{a}}$, K. Peter R. Nilsson ${ }^{\mathrm{b}}$, Katarina Kågedal ${ }^{\mathrm{c}}$ and Ann-Christin Brorsson ${ }^{\mathrm{a}}$

${ }^{\mathrm{a} D i v i s i o n}$ of Molecular Biotechnology, Department of Physics, Chemistry and Biology, Linköping University, Sweden.

${ }^{\mathrm{b}}$ Division of Organic Chemistry, Department of Physics, Chemistry and Biology, Linköping University, Sweden.

${ }^{\mathrm{c}}$ Department of Clinical and Experimental Medicine, Linköping University, Sweden.

\section{E-mail addresses}

Anna-Lena Göransson: anngo@ifm.liu.se

K. Peter R. Nilsson: petni@ifm.liu.se

Katarina Kågedal: katarina.kagedal@liu.se

\section{Corresponding author}

Ann-Christin Brorsson, Division of Molecular Biotechnology, Department of Physics, Chemistry and Biology, Linköping University, SE-581 83, Linköping, Sweden:

Fax: +46 13-281399

Tel: +46-13-286648

E-mail: anki@ifm.liu.se 


\begin{abstract}
The formation of amyloid- $\beta$ peptide $(\mathrm{A} \beta)$ aggregates at an early stage during the selfassembly process is an important factor in the development of Alzheimer's disease. The toxic effect is believed to be exerted by prefibrillar species of $A \beta$. It is therefore important to identify which prefibrillar species are toxic and characterize their distinct properties. In the present study, we investigated the in vitro aggregation behavior of $A \beta$-derived peptides possessing different levels of neurotoxic activity, using fluorescence spectroscopy in combination with transmission electron microscopy. The toxicity of various $A \beta$ aggregates was assessed by using cultures of human neuroblastoma cells. Through combined use of the fluorescence probe 8-anilino-1-napthalenesulfonate (ANS) and the novel luminescent probe pentamer formyl thiophene acetic acid (p-FTAA), we were able to identify those A $\beta$ peptidederived prefibrillar species which exhibited cellular toxicity. In particular, species, which formed early during the aggregation process and showed strong p-FTAA and ANS fluorescence, were the species that possessed toxic activities. Moreover, by manipulating the aggregation conditions, it was possible to change the capacity of the $\mathrm{A} \beta$ peptide to form nontoxic versus toxic species.
\end{abstract}

\title{
Keywords
}

Alzheimer's disease; amyloid- $\beta$ peptide; fluorescence spectroscopy; cell toxicity; protein aggregation

\section{Abbreviations}

AD, Alzheimer's disease; A $\beta$, amyloid- $\beta$ peptide; APP, amyloid precursor protein; TEM, transmission electron microscopy; ANS, 8-anilino-1-napthalensulfonate; ThT, thioflavin T; $\mathrm{p}$ FTAA, pentamer formyl thiophene acetic acid; pFTAA $^{+}$species, species recognized by pFTAA; ThT $^{+}$species, species recognized by ThT. 


\section{Introduction}

Misfolded proteins are involved in several diseases, e.g., Huntington's disease, Parkinson's disease, Creutzfeldt-Jakob disease and Alzheimer's disease (AD) [1]. These disorders are associated with the presence of protein deposits comprising amyloid fibrils. It is important to increase our understanding of the molecular processes involved in misfolding and aggregation of proteins in order to understand how these events are connected to the pathogenesis of diseases. The aggregation of the $A \beta$ peptide into plaques has long been considered the key neuropathological hallmark of $\mathrm{AD}[2]$. $\mathrm{A} \beta$ peptides are produced in the brain by proteolytic cleavage of the amyloid precursor protein (APP) by $\beta$ - and $\gamma$-secretases [3]. Depending on the site of cleavage, different sized $A \beta$ peptides are generated, with $A \beta_{40}$ and $A \beta_{42}$ being the most commonly occurring $A \beta$ isoforms. $A \beta_{42}$ has a higher propensity to form prefibrillar and fibrillar aggregates than $A \beta_{40}$ and has also been reported to be more toxic [4]. Furthermore, it has been shown that an increased ratio of $A \beta_{42}$ to $A \beta_{40}$ correlates with an increased risk of subsequently developing $\mathrm{AD}$ [5]. Monomeric $\mathrm{A} \beta$ aggregates into insoluble fibrils via different soluble intermediates [6]. To date, the main consensus is that early formed oligomeric intermediates (prefibrillar species) are the critical entities which cause cellular damage, and they may be the key effectors of pathogenicity in AD [7]. Thus, it is important to identify which prefibrillar species are directly involved in cellular damage and determine their distinct biophysical properties.

Like many of the diseases associated with misfolded proteins, AD has both sporadic and inherited forms. One example of familial $A D$ is the mutation E22G of $A \beta_{42}$ (also known as the Arctic mutation), which causes early onset of the disease [8]. The Arctic mutation accelerates the protein aggregation process and, in particular, accelerates the formation of prefibrillar species [8], further supporting the notion that these early formed prefibrillar species exert the neurotoxic activity. In recent studies, we have shown that the Arctic peptide is more neurotoxic than $A \beta_{42}$ when expressed in the central nervous system of Drosophila melanogaster $[4,9]$. It was also found that a single-point mutation, I31E, introduced into the Arctic peptide (henceforth denoted the Rescue variant), abolishes the pathogenicity by significantly reducing the propensity to form prefibrillar aggregates.

In the present study, we investigated how differences in toxic behavior between $A \beta_{42}$, Arctic $\left(\mathrm{A} \beta_{42} \mathrm{E} 22 \mathrm{G}\right)$ and Rescue $\left(\mathrm{A} \beta_{42} \mathrm{E} 22 \mathrm{G} / \mathrm{I} 31 \mathrm{E}\right)$ related to the propensity of these peptides to form different protein aggregates by characterizing the in vitro aggregation behavior of these variants using fluorescent probes and fluorescence spectroscopy in combination with cell toxicity studies and transmission electron microscopy (TEM). The formation of amyloid fibrils was essentially probed by thioflavin T (ThT) fluorescence [10], and the hydrophobic properties of aggregated species were investigated using ANS fluorescence [11]. A novel luminescent probe, p-FTAA, was used to identify species which were not efficiently recognized by ThT, since it has been shown earlier that p-FTAA is a useful probe for detecting prefibrillar non-thioflavinophilic protein aggregates [12]. The aim was to carefully decipher the biophysical properties of aggregated species generated by the three selected peptides and identify the prefibrillar structures that are associated with neurodegeneration.

\section{Materials and methods}

\subsection{Preparation of the A $\beta$ peptides}

Synthetic $A \beta_{42}, A \beta_{42} E 22 G$ and $A \beta_{42}$ E22G/I31E (Bachem, Weil am Rhein, Germany) were dissolved in trifluoroacetic acid and sonicated on ice for 20s. The trifluoroacetic acid was removed by lyophilization, the peptide was dissolved in 1,1,1,3,3,3-hexafluoro-2-propanol 
and then divided into aliquots. The 1,1,1,3,3,3-hexafluoro-2-propanol was removed by rotary evaporation at room temperature, and the quantity of peptide in the aliquots was determined with the Bradford $D C$ protein Assay (Bio-Rad Laboratories, Hemel Hempstead, UK).

\subsection{Fluorescence measurements}

$\mathrm{A} \beta$ samples were dissolved to a concentration of $20 \mu \mathrm{M}\left(\mathrm{A} \beta_{42}\right)$ or $30 \mu \mathrm{M}\left(\mathrm{A} \beta_{42} \mathrm{E} 22 \mathrm{G}\right.$ and $\left.\mathrm{A} \beta_{42} \mathrm{E} 22 \mathrm{G} / \mathrm{I} 31 \mathrm{E}\right)$ in $50 \mathrm{mM} \mathrm{Na} \mathrm{PO}_{4}(\mathrm{pH}$ 7.4). The fluorescence probes ThT, p-FTAA and ANS were used at concentrations of $0.2 \mu \mathrm{M}$ (p-FTAA), $0.5 \mu \mathrm{M}$ (ThT), $100 \mu \mathrm{M}$ (ANSmeasurements of $A \beta_{42}$ ) and $50 \mu \mathrm{M}$ (ANS-measurements of $A \beta_{42} E 22 G$ and $A \beta_{42} E 22 \mathrm{G} / \mathrm{I} 31 \mathrm{E}$ ). Samples were incubated under quiescent conditions at $37^{\circ} \mathrm{C}$ in a Tecan Safire 2 plate reader. Peptide solutions of $A \beta_{42}$ E22G and $A \beta_{42}$ E22G/I31E, with a concentration of $20 \mu \mathrm{M}$, were also incubated at $30{ }^{\circ} \mathrm{C}$ under shaking conditions. Fluorescence intensities were recorded by excitation at $375 \mathrm{~nm}$ (ANS) or $440 \mathrm{~nm}$ (ThT and p-FTAA), and emission spectra were recorded between $470 \mathrm{~nm}$ and $550 \mathrm{~nm}$. The fluorescence profile data presented were obtained from selected curves, representing at least three replicates.

\subsection{Transmission electron microscopy}

Carbon-coated nickel grids were charged with UV light for $5 \mathrm{~min}$. Ten microliters of the peptide sample was dispensed onto the grid. After $2 \mathrm{~min}$, the liquid was removed by blotting with a filter paper. The grid was washed with two aliquots of $10 \mu \mathrm{l} \mathrm{dH}_{2} \mathrm{O}$. Staining was performed using $10 \mu 12 \%$ uranyl-acetate for $2 \mathrm{~min}$, and then the grid was blotted dry. The dry grid was stored in a grid box until it was viewed with a JEOL 1230 TEM (Jeol Ltd.; Tokyo, Japan) equipped with an ORIUS SC 1000 CCD camera.

\subsection{Cell culture and viability measurement}

$\mathrm{A} \beta$ samples were dissolved to a concentration of $30 \mu \mathrm{M}$ in $50 \mathrm{mM} \mathrm{Na} 2 \mathrm{PO}_{4}(\mathrm{pH} 7.4)$ and incubated at $37{ }^{\circ} \mathrm{C}$. Human SH-SY5Y neuroblastoma cells were cultured in Minimum Essential Medium (MEM) Glutamax (Invitrogen, Paisley, UK), which was supplemented with $10 \%$ calf serum (PAA Laboratories, Pasching, Austria), $2 \mathrm{mM}$ glutamine, $50 \mathrm{IU} / \mathrm{ml}$ penicillin$\mathrm{G}$ and $50 \mathrm{mg} / \mathrm{ml}$ streptomycin (Lonza, Bazel, Switzerland). Prior to experiments, the cells were differentiated by treating them with $10 \mu \mathrm{M}$ retinoic acid (Sigma Aldrich, St. Louis, MO, USA) for 7 days. The cells were trypsinized and seeded in culture plates at a density of 90,000 cells $/ \mathrm{cm}^{2}$ and subsequently exposed to $3 \mu \mathrm{M} \mathrm{A} \beta$ in serum-free medium for 6 days. After treatment, cell cultures were morphologically examined in a phase contrast microscope and viability was measured using XTT (Roch, Germany) according to instructions from the company.

\subsection{Statistical analysis}

Statistical significance was determined using the Kruskal-Wallis multiple comparison test followed by the Mann-Whitney U-test. Differences were considered significant when $\mathrm{p} \leq$ 0.005 .

\section{Results and discussion}

\subsection{Aggregation of $A \beta_{42}$}


We first examined fibril formation of $A \beta_{42}$ to compare the aggregation behavior of this peptide with the more toxic Arctic variant and the essentially nontoxic Rescue variant [9]. Fig. 1A shows the aggregation of the $A \beta_{42}$ peptide probed by ThT, p-FTAA and ANS fluorescence under quiescent incubation. At the start of the aggregation process, species were formed that were recognized by all three probes. Comparison of the p-FTAA and ThT fluorescence profiles showed that the p-FTAA signal increased more rapidly than the ThT signal and reached a maximum value after five hours followed by a slow decrease. In contrast, the ThT fluorescence signal increased continuously throughout the experiment. These observations suggest that efficient fluorescence from p-FTAA or ThT were achieved when different aggregated $A \beta$ species were present during the aggregation process. Initially, one type of species which gave rise to strong emission from p-FTAA (these species are henceforth denoted p-FTAA ${ }^{+}$) formed rapidly, giving rise to a rapid increase in the p-FTAA signal. Another type of species which yielded strong ThT fluorescence (these species are henceforth denoted $\mathrm{ThT}^{+}$) formed more slowly as shown by the gradual increase in the ThT signal. TEM images at the end of the aggregation experiment confirmed fibril formation in the presence of both probes (Fig. 1B and C). Indeed, the morphologies of the fibrils formed in the presence of ThT and p-FTAA were very similar, comprising predominantly of twisted fibrils of $\mu \mathrm{m}$ length scale. The results suggest that the p-FTAA fluorescence curve initially reflects the rapid formation of $\mathrm{p}-\mathrm{FTAA}^{+}$prefibrillar species, which are later converted to mature twisted $\mathrm{ThT}^{+}$ fibrils, as reflected by the steady decrease in the p-FTAA fluorescence and increase in the ThT fluorescence observed after five hours and during the remaining timepoints in the experiment. In agreement with the p-FTAA fluorescence profile, a rapid increase in the ANS fluorescence signals was also observed, indicating that the p-FTAA ${ }^{+}$species formed had exposed hydrophobic surfaces. TEM images recorded during this increase revealed a mixture of globular structures (Fig. 1D, black arrow), filaments of various lengths and long fibrils (Fig. 1D, dashed black arrow and white arrow, respectively). After five hours, when the pFTAA fluorescence had started to decrease, the ANS signal continued to show a small and steady increase during the remaining time of the experiment, in agreement with the ThT fluorescence profile. Thus, both the p-FTAA ${ }^{+}$prefibrillar species, which formed at the start of aggregation, and the mature $\mathrm{ThT}^{+}$fibrils, which accumulated by the end of the experiment, had exposed hydrophobic surfaces.
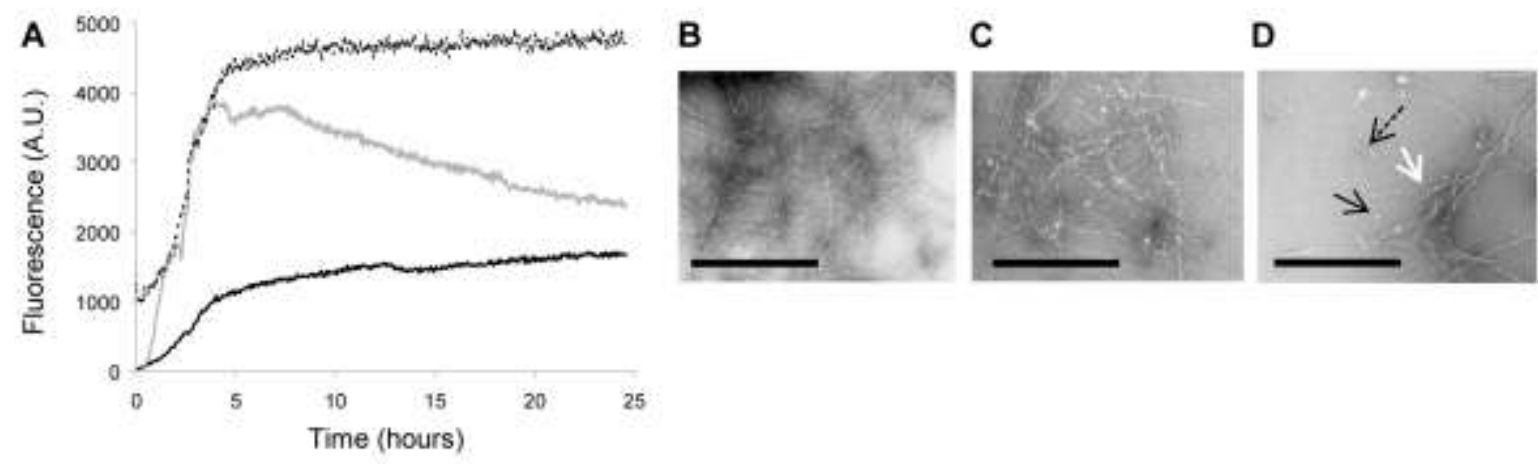

Fig. 1. Aggregation of $A \beta_{42}$ under quiescent incubation probed by ThT, p-FTAA and ANS fluorescence in combination with TEM. The progression of the aggregation probed by ThT (black line), p-FTAA (grey line) and ANS (dotted line) (A). TEM images of peptide samples taken at the end of the aggregation experiments in the presence of ThT (B) and p-FTAA (C). TEM image of peptide sample incubated without probes after $2.5 \mathrm{~h}$ (D). Black arrow indicates globular structures, dashed black arrow indicates filaments and white arrow indicates fibrils. Scale bar $=500 \mathrm{~nm}$. 


\subsection{Characterization of the aggregation process for Arctic and Rescue under quiescent conditions}

When probing the aggregation process of Arctic using ThT, p-FTAA and ANS, a fluorescence signal from both ANS and p-FTAA was already observed at the start of the experiment, indicating that $\mathrm{p}-\mathrm{FTAA}^{+}$hydrophobic prefibrillar species appeared immediately after dissolving the peptide in the buffer (Fig. 2A). During the aggregation process, the pFTAA signal reached a maximum value at $15 \mathrm{~h}$, followed by a gradual decrease, whereas the ANS signal increased in parallel to the p-FTAA signal for the first $15 \mathrm{~h}$ and then continued to increase during the remainder of the experiment. The ThT fluorescence signal gradually increased from the start of aggregation to the end of the experiment. Overall, the Arctic fluorescence profiles showed similarities to the $A \beta_{42}$ fluorescence profiles, where the early formed p-FTAA ${ }^{+}$hydrophobic prefibrillar species were later converted into $\mathrm{ThT}^{+}$matured amyloid fibrils with exposed hydrophobic surfaces.

The Rescue aggregation profiles, probed by ThT, p-FTAA and ANS showed a lag phase followed by a growth phase ending with a plateau (Fig. 2B). Here, the magnitudes observed for the fluorescence signals differed dramatically; the p-FTAA signals were very low compared to the ThT and ANS signals. Apparently, the Rescue peptide is able to form aggregates that give rise to strong ThT and ANS fluorescence but less fluorescence from pFTAA. The presence of a lag phase in the p-FTAA and ANS profiles of the Rescue peptide implies that if p-FTAA ${ }^{+}$hydrophobic prefibrillar species were formed, they must exist at a very low concentration, which, in combination with the late onset of ThT fluorescence, indicates that the initial formation of fibrillar species occurred from a low concentration pool of prefibrillar species. The parallel increase in the ThT and ANS fluorescence profiles demonstrates that the $\mathrm{ThT}^{+}$species formed had exposed hydrophobic surfaces.

TEM images taken at the end of the aggregation experiments show the morphology of aggregates formed in the presence of ThT, p-FTAA and ANS for both Arctic (Fig. 2C-E) and Rescue (Fig. 2F-H). In line with previous observation [9], a large amount of prefibrillar species were detected for the Arctic peptide, including globular structures (Fig. 2C and D, black arrows) and short curvilinear filaments (Fig. 2C-E, dashed black arrows) together with long fibrils (Fig. 2C-E, white arrows). The large quantity of prefibrillar species accumulated for the Arctic peptide was most likely responsible for the distinct p-FTAA signal intensities found for this peptide. The p-FTAA profile of the Arctic peptide showed that the amount of prefibrillar species reached a maximum at around $15 \mathrm{~h}$, followed by a decrease in the pool of prefibrillar species, of which a fraction was converted into more mature fibrils. The residual p-FTAA fluorescence observed at later times was most probably due to the remaining prefibrillar species, which can be seen in the TEM image (Fig. 2D). The observed fibrils gave rise to the ThT signal, which increased over time in the Arctic ThT profile (Fig. 2A and C). In contrast to the Arctic peptide, the Rescue peptide only gave an accumulation of mature fibrils with a twisted appearance (Fig. 2F-H), which were able to form intertwined structures composed of several fibrils (Fig. 2F, inset), and no prefibrillar species were observed in the samples. The absence of prefibrillar species in the TEM images supports the fluorescence data, where no p-FTAA ${ }^{+}$prefibrillar species were detected. As for $A \beta_{42}$ and Arctic, the mature Rescue fibrils were well recognized by ThT and ANS (Fig. 3B, F and H). 


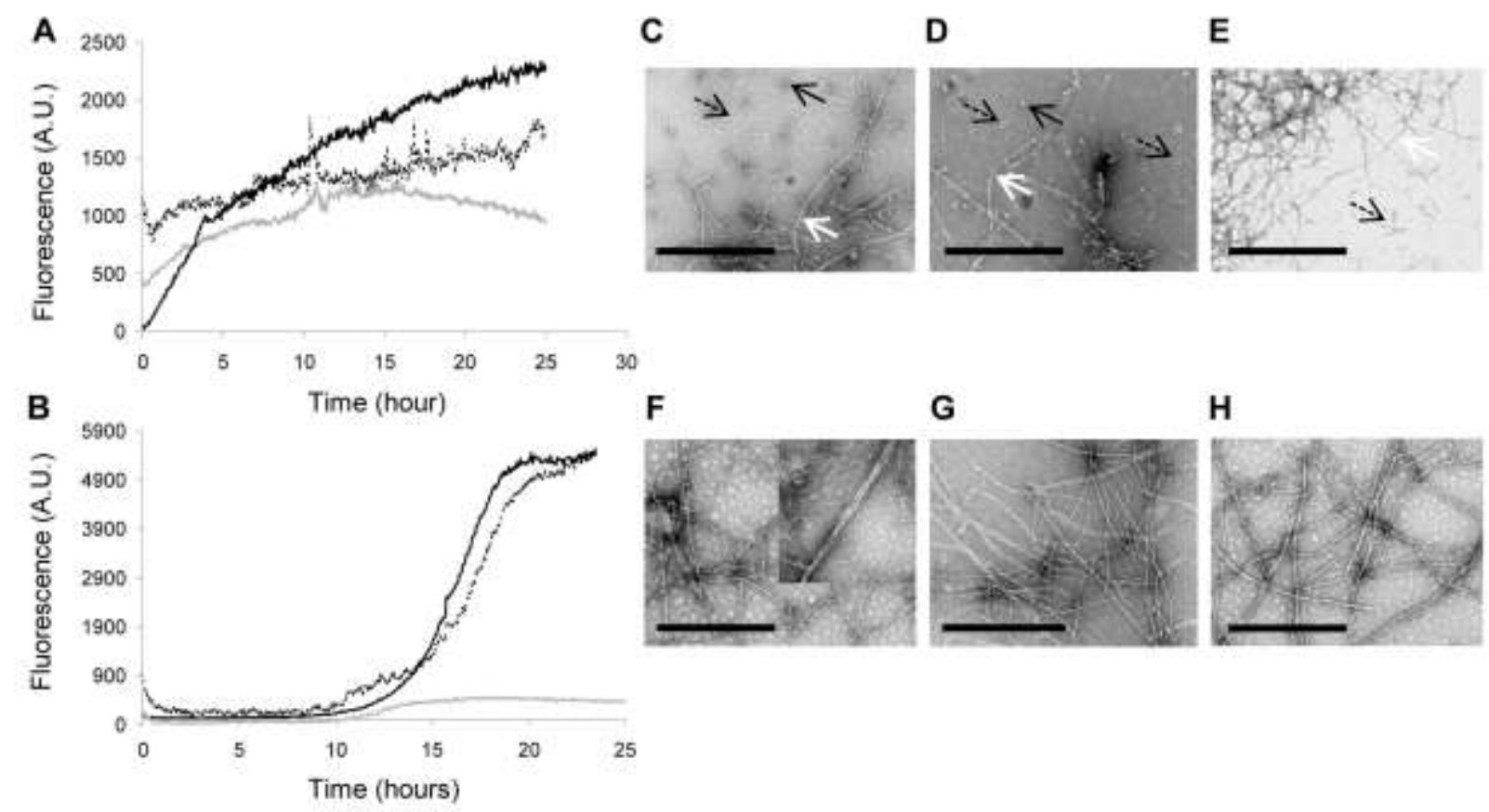

Fig. 2. Aggregation of Arctic and Rescue under quiescent incubation probed by ThT, p-FTAA and ANS fluorescence in combination with TEM. Aggregation of Arctic (A) and Rescue (B) probed by ThT (black line), p-FTAA (grey line) and ANS (dotted line). TEM images recorded at the end of the aggregation experiments of Arctic aggregates in the presence of ThT $(\mathrm{C}), \mathrm{p}-$ FTAA (D) and ANS (E) and of Rescue aggregates in the presence of ThT (F), p-FTAA (G) and ANS (H). Black arrows indicate globular structures, dashed black arrows indicate short filaments and white arrows indicate long fibrils. Scale bar $=500 \mathrm{~nm}$.

\subsection{Characterization of the aggregation process for Arctic and Rescue under shaken conditions}

Stirring and shaking protein samples during aggregation can affect amyloid fibril formation and change the properties of the resulting aggregated species [13]. Therefore, we next examined the aggregation properties of Arctic and Rescue under conditions in which the samples were shaken. At the start of the aggregation process for the Arctic peptide, species were formed which were recognized by ThT, p-FTAA and ANS (Fig. 3A), reflecting the formation of $\mathrm{ThT}^{+}$and $\mathrm{p}-\mathrm{FTAA}^{+}$aggregates with exposed hydrophobic patches. After reaching a maximum fluorescence value after only $30 \mathrm{~min}$, both the p-FTAA and ANS signals then declined during the remaining timepoints. The maximum fluorescence value of the ThT signal was reached after around $5 \mathrm{~h}$ and then remained fairly constant during the remainder of

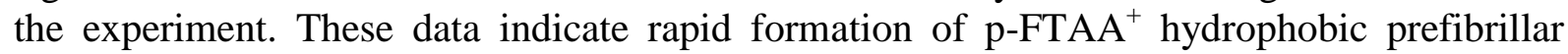
species, which are later converted to $\mathrm{ThT}^{+}$species with exposed hydrophobic surfaces as revealed by the significant ANS signal at the end of the experiment.

A TEM image recorded at the end of the aggregation experiment shows the accumulation of filaments with various lengths (Fig. 3C, dashed black arrows) along with more amorphous assemblies in the presence of ThT. A TEM image of accumulated aggregates at the end of the experiment in the presence of p-FTAA shows the presence of short filaments, which were stacked together (Fig. 3D). These species showed no increase in fluorescence from p-FTAA since the p-FTAA signal obtained at the end of the experiment was relatively low (Fig. 3A). The aggregates that accumulated in the presence of ANS under shaken conditions were rather 
amorphous (Fig. 3E). The results suggest that these structures contain exposed hydrophobic patches, which allow ANS to bind.

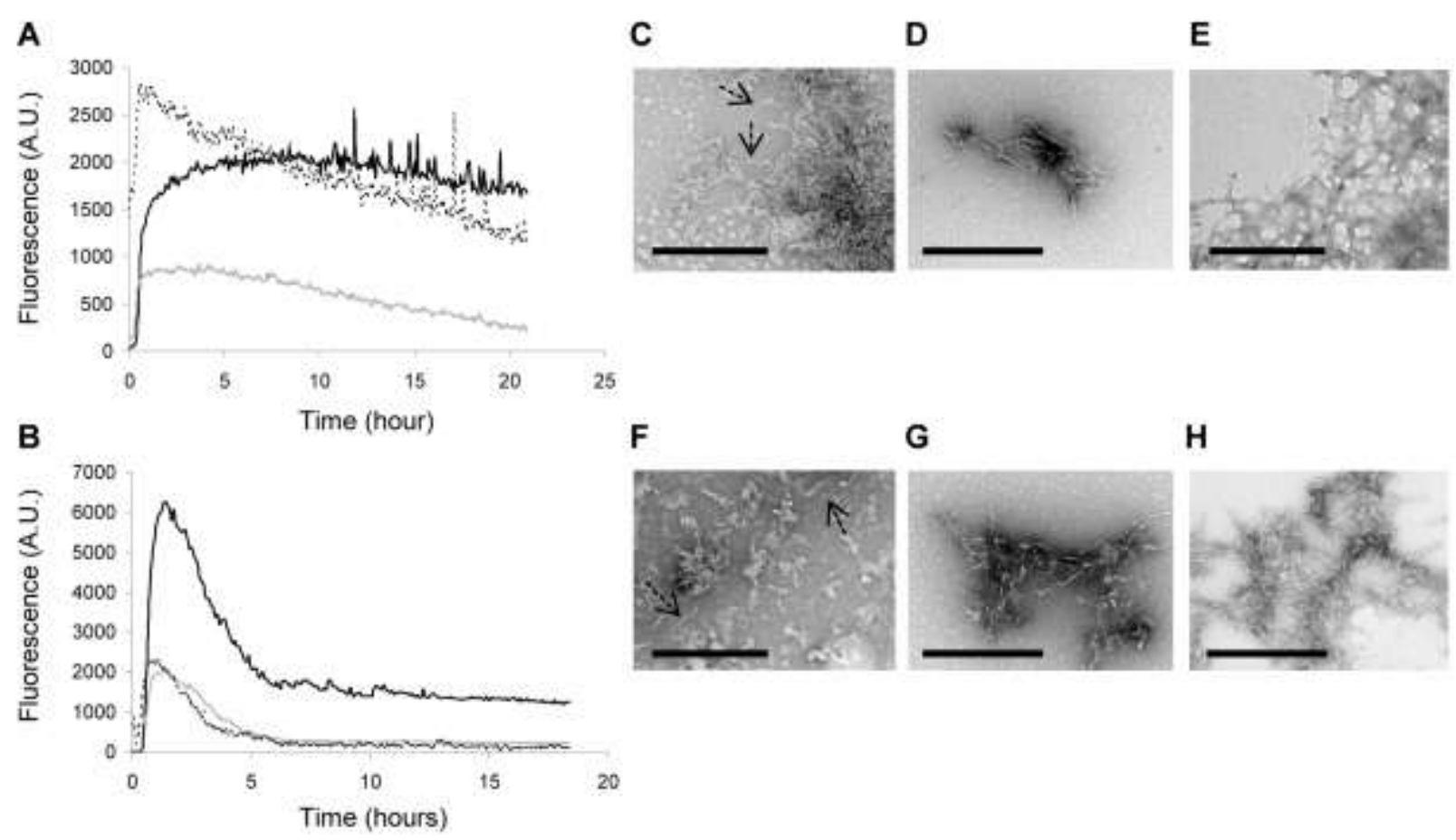

Fig. 3. Aggregation of Arctic and Rescue under shaken conditions probed by ThT, p-FTAA and ANS fluorescence in combination with TEM. Aggregation of Arctic (A) and Rescue (B) probed by ThT (black line), p-FTAA (grey line) and ANS (dotted line). TEM images recorded at the end of the aggregation experiments of Arctic aggregates in the presence of ThT (C), pFTAA (D) and ANS (E) and of Rescue aggregates in the presence of ThT (F), p-FTAA (G) and ANS (H). Dashed black arrows indicate filaments of various lengths. Scale bar $=500 \mathrm{~nm}$.

The Rescue peptide initially formed species very rapidly, which were recognized by ThT, pFTAA and ANS (Fig. 3B). Thus, in contrast to quiescent conditions, the Rescue peptide formed both prefibrillar and fibrillar species under shaken conditions. However, the signals due to all three probes declined very quickly and reached a steady level after about $5 \mathrm{~h}$. At the end of the experiment, only a low signal due to ThT was observed. Thus, the early formed $\mathrm{ThT}^{+}$and $\mathrm{p}-\mathrm{FTAA}^{+}$aggregates containing hydrophobic patches were rapidly converted to aggregates that showed no fluorescence from p-FTAA and ANS and only to small extent fluorescence from ThT. A TEM image taken at the end of the aggregation experiment shows the accumulation of short filaments together with more amorphous assemblies in the presence of ThT (Fig. 3F, dashed black arrows). Assemblies accumulated in the presence of p-FTAA consisted of short and thin filaments that were stacked together (Fig. 3G), whereas the filaments accumulated in the presence of ANS were somewhat thicker (Fig. 3H).

\subsection{Cell toxicity of aggregated species}

To elucidate the toxicity of different aggregated species, their effects on cellular viability were examined. In parallel, the peptide samples were examined by TEM. We found that species that formed immediately after dissolving the Arctic peptide in the buffer $(0 \mathrm{~h}$ of incubation) decreased cellular viability to about $40 \%$ of the control (Fig. 4A and B). A TEM image captured immediately after dissolving the Arctic peptide in the buffer revealed the 
presence of globular species of different sizes together with larger amorphous aggregates (Fig. $4 \mathrm{C}$, black and dashed white arrows, respectively). After incubation of the peptide for $30 \mathrm{~h}$ under quiescent conditions, short curvilinear filaments were found to be the dominant species in the sample (Fig. 4E, dashed black arrows) along with a few more well-defined fibrils (Fig. $4 \mathrm{E}$, white arrow). These species could not be linked to cell toxicity since no significant reduction in cellular viability was detected (Fig. 4A and D).

Surprisingly, we found that the Rescue peptide, which does not exert any significant toxicity when expressed in Drosophila [4,9], was able to induce cell death when aggregated under defined conditions. Specifically, aggregates that formed after $1 \mathrm{~h}$ under shaken conditions decreased the cellular viability to about $60 \%$ of the control (Fig. 4A and F). A TEM image captured after $1 \mathrm{~h}$ under shaken conditions reveals the formation of a substantial amount of prefibrillar species, including small globular and thin filamentous structures together with a few fibrils (Fig. 4G, black, dashed black and white arrows, respectively). A TEM image of species formed immediately after dissolving the peptide shows a small amount of tiny globular species and amorphous aggregates (Fig. 4I, black and dashed white arrow, respectively), whereas long mature fibrils were observed after incubation of the peptide for 30 $\mathrm{h}$ under quiescent conditions (Fig. 4K). These species could not be linked to cell toxicity since no significant reduction in cellular viability was detected for these samples (Fig. 4A, H and J).

The cell toxicity data clearly reveal that the Rescue peptide is able to form cytotoxic aggregates after $1 \mathrm{~h}$ under shaken conditions. Interestingly, the corresponding TEM image at this timepoint shows the presence of a substantial amount of prefibrillar species (Fig. 4G). Moreover the fluorescence profiles for the aggregation of the Rescue peptide under shaken conditions reveal the presence of early formed species that gave rise to p-FTAA and ANS fluorescence (Fig. 3B). Consequently, the p-FTAA ${ }^{+}$hydrophobic prefibrillar species that were formed under these conditions seem to be responsible for the cell toxicity mediated by the Rescue peptide.

\subsection{In vivo and in vitro toxicity directly correlate with distinct physiochemical properties of the prefibrillar species}

It is apparent that there is a clear link between neurodegenerative properties of the $A \beta$ peptide in the central nervous system of Drosophila and the propensity of the peptide to form prefibrillar assemblies at an early stage of the aggregation process. Importantly, these prefibrillar species are characterized by strong fluorescence from p-FTAA and by having exposed hydrophobic surfaces as probed by ANS. The studied peptides were chosen because they have been shown to have different effects on fly longevity when expressed in the central nervous system of $D$. melanogaster [4,9]. In those studies, it was found that the Arctic peptide causes severe neurodegeneration, which manifested as a $74 \%$ reduction in longevity compared to flies expressing the nontoxic $A \beta_{40}$ peptide. The $A \beta_{42}$ peptide reduced the longevity by $36 \%$, whereas no significant signs of neurodegeneration were observed for the Rescue peptide. The results of the present study show that under quiescent conditions, the two neurotoxic $A \beta$ peptides $A \beta_{42}$ and Arctic form p-FTAA ${ }^{+}$prefibrillar species with exposed hydrophobic surfaces at the start of the aggregation process. Under these conditions, the nontoxic Rescue peptide did not form prefibrillar species that resulted in fluorescence from $\mathrm{p}$ FTAA or ANS, nor did it form aggregates that could induce cell death. Taken together, these findings strongly suggest that the observed p-FTAA $^{+}$hydrophobic prefibrillar species are responsible for the neurotoxic effect of $A \beta_{42}$ and Arctic seen both in the cell- and in the Drosophila model. Interestingly, by performing the aggregation of the Rescue peptide under shaken conditions species were formed that gave rise to p-FTAA and ANS fluorescence and 


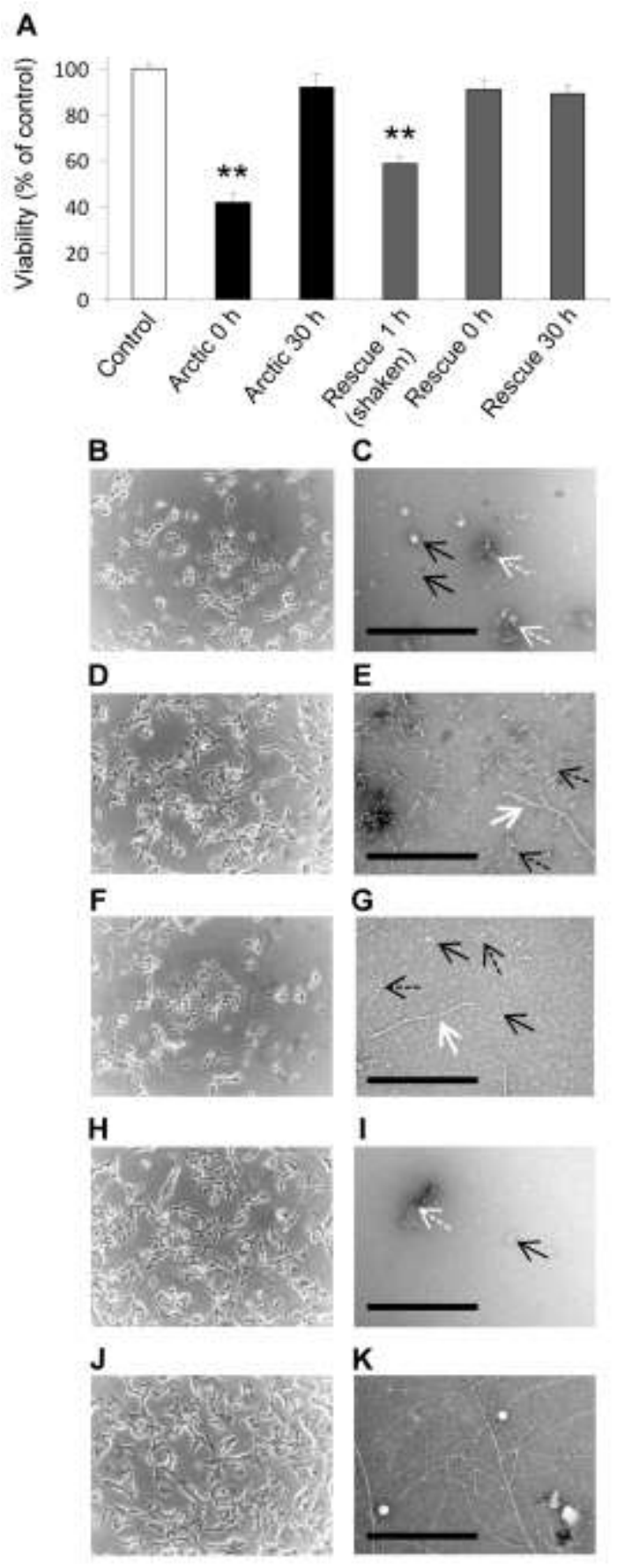

Fig. 4. Cell toxicity and TEM images of different A $\beta$ aggregates. (A) Viability of human SH-SY5Y neuroblastoma cells after exposure to $A \beta$ using the XTT viability assay. Viability is expressed as percentage of untreated cultures (control), $n=3$. Images of cells after exposure to aggregates of the Arctic peptide formed at $0 \mathrm{~h}$ (B) and after $30 \mathrm{~h}$ (D) incubation under quiescent conditions. Images of cells after exposure to aggregates of the Rescue peptide formed after $1 \mathrm{~h}(\mathrm{~F})$ incubation under shaking conditions, and at $0 \mathrm{~h}(\mathrm{H})$ and after $30 \mathrm{~h}(\mathrm{~J})$ incubation under quiescent conditions. TEM images of the Arctic peptide at $0 \mathrm{~h}(\mathrm{C})$ and after $30 \mathrm{~h}(\mathrm{E})$ incubation under quiescent conditions. TEM images of the Rescue peptide after $1 \mathrm{~h}(\mathrm{G})$ incubation under shaking conditions, and at $0 \mathrm{~h}(\mathrm{I})$ and after $30 \mathrm{~h}(\mathrm{~K})$ incubation under quiescent conditions. Black arrows indicate globular species, dashed white arrows indicate amorphous aggregates, dashed black arrows indicate short filaments and white arrows indicate fibrils. Scale bar $=500 \mathrm{~nm}$. Asterisks indicate significant decreased cellular viability. $* * P \leq 0.005$. 
were able to induce cell death. Thus, the cell toxicity is clearly dependent on formation of defined p-FTAA $^{+}$hydrophobic prefibrillar species. The neurotoxic properties of the A $\beta$ peptide must be linked to the propensity of the peptide to form aggregates that possess certain properties in terms of size and biophysical parameters which are required for cell toxicity. It has previously been suggested that the exposure of hydrophobic surfaces is crucial for the toxicity of protein oligomers and of $A \beta$ aggregates promoting interference of the aggregates with membrane structures $[14,15]$. Our results strongly support these findings and, in addition, show that p-FTAA can be used to probe early formed prefibrillar species that are linked to cell toxicity.

\section{Acknowledgments}

We thank Prof. Bengt-Harald Jonsson for valuable discussions and Mildred Otieno and Daniel Kanmert for technical assistance. This work was supported by the Swedish National Graduate School in Science, Technology and Mathematics Education Research (FontD) (A.-L.G.), the Swedish Alzheimer's Foundation and by Söderberg foundation (A.-C.B.).

\section{References}

[1] C.M. Dobson, The structural basis of protein folding and its links with human disease, Philos. Trans. R. Soc. Lond. B Biol. Sci. 356 (2001) 133-145.

[2] J.A. Hardy, G.A. Higgins, Alzheimer's disease: the amyloid cascade hypothesis, Science 256 (1992) 184-185.

[3] M.S. Wolfe, S.Y. Guenette, APP at a glance, J. Cell. Sci. 120 (2007) 3157-3161.

[4] L.M. Luheshi, G.G. Tartaglia, A.-C. Brorsson, et al., Systematic in vivo analysis of the intrinsic determinants of amyloid $\beta$ pathogenicity, PLoS Biol. 5 (2007) e290.

[5] S. Kumar-Singh, J. Theuns, B. Van Broeck, et al., Mean age-of-onset of familial Alzheimer disease caused by presenilin mutations correlates with both increased $A \beta 42$ and decreased A $\beta 40$, Hum. Mutat. 27 (2006) 686-695.

[6] D.M. Walsh, A. Lomakin, G.B. Benedek, et al., Amyloid $\beta$-protein fibrillogenesis. Detection of a protofibrillar intermediate, J. Biol. Chem. 272 (1997) 22364-22372.

[7] J.P. Cleary, D.M. Walsh, J.J. Hofmeister, et al., Natural oligomers of the amyloid- $\beta$ protein specifically disrupt cognitive function, Nat. Neurosci. 8 (2005) 79-84.

[8] C. Nilsberth, A. Westlind-Danielsson, C.B. Eckman, et al., The 'Arctic' APP mutation (E693G) causes Alzheimer's disease by enhanced A $\beta$ protofibril formation, Nat. Neurosci. 4 (2001) 887-893.

[9] A.-C. Brorsson, B. Bolognesi, G.G. Tartaglia, et al., Intrinsic determinants of neurotoxic aggregate formation by the amyloid $\beta$ peptide, Biophys. J. 98 (2010) 1677-1684.

[10] H. LeVine III, W. Ronald, Quantification of $\beta$-sheet amyloid fibril structures with thioflavin T, Methods Enzymol. (1999) 274-284.

[11] L. Stryer, The interaction of a naphthalene dye with apomyoglobin and apohemoglobin: A fluorescent probe of non-polar binding sites, J. Mol. Biol. 13 (1965) 482-495.

[12] P. Hammarström, R. Simon, S. Nyström, et al., A fluorescent pentameric thiophene derivative detects in vitro-formed prefibrillar protein aggregates, Biochemistry 49 (2010) 6838-6845.

[13] W.-F. Xue, A.L. Hellewell, W.S. Gosal, et al., Fibril fragmentation enhances amyloid cytotoxicity, J. Biol. Chem. 284 (2009) 34272-34282.

[14] B. Bolognesi, J.R. Kumita, T.P. Barros, et al., ANS binding reveals common features of cytotoxic amyloid species, ACS Chem. Biol. 5 (2010) 735-740. 
[15] S. Campioni, B. Mannini, M. Zampagni, et al., A causative link between the structure of aberrant protein oligomers and their toxicity, Nat. Chem. Biol. 6 (2010) 140-147. 